\title{
ON THE COVERING OF POLYHEDRA BY POLYHEDRA ${ }^{1}$
}

\section{A. J. HOFFMAN}

1. Introduction. A polyhedron $P$ is the intersection of a finite number of closed half-spaces of a finite-dimensional Euclidean space. We do not exclude the possibility that $P$ is empty or that $P$ is the whole space. Suppose a polyhedron $P$ is covered by a finite number of closed convex sets $C_{1}, \cdots, C_{k}$. Then it is intuitively plausible that, if one or more of the $C_{j}$ is "round" (i.e., not a polyhedron), then each $C_{j}$ could be replaced by a subpolyhedron and $P$ would still be covered. The purpose of this note is to verify this intuition, and to show its relevance to a class of sufficient conditions for a rectangular matrix to be of full rank.

We are very grateful to Michael Rabin, both for proposing the problem and for other valuable conversations on related topics.

2. Theorem. Let $C_{1}, \cdots, C_{k}$ be closed convex sets, $P$ a polyhedron, $P=C_{1} \cup \ldots \cup C_{k}$. Then there exist polyhedra $P_{j} \subset C_{j}, j=1, \cdots, k$ such that $P=P_{1} \cup \cdots \cup P_{k}$.

Proof. We prove the theorem by induction on $\operatorname{dim} P$ (i.e., the dimension of the lowest dimensional flat containing $P$ ). The theorem obviously holds if $\operatorname{dim} P=0$ or 1 , so assume $\operatorname{dim} P=n$ and that the theorem has been proved for all $P$ such that $\operatorname{dim} P<n$.

In the argument that follows we shall make use of the following well-known facts:

(2.1) The convex hull of a finite number of polyhedra is a polyhedron;

(2.2) If $Q$ and $R$ are polyhedra, the closure of $Q-R$ is the union of a finite number of polyhedra.

Our first step is to partition the space $R^{n}$ containing $P$ into closed orthants $Q_{1}, \cdots, Q_{2^{n}}$. Clearly each $P \cap Q_{j}=\left(C_{1} \cap Q_{j}\right) \cup \ldots$ $\cup\left(C_{k} \cap Q_{j}\right), j=1, \cdots, 2^{n}$. If we prove the theorem for each $P \cap Q_{j}$, then the theorem for $P$ follows from (2.1). Hence we may assume $P$ contained in a closed orthant, which we may take without loss of generality to be the first orthant. Therefore

$$
x=\left(x_{1}, \cdots, x_{n}\right) \in P \quad \text { implies } \quad x_{j} \geqq 0, \quad j=1, \cdots, n .
$$

Received by the editors October 15, 1968.

1 The research reported was sponsored in part by the Office of Naval Research under Contract Nonr-3775(00). 
By the definition of $\operatorname{dim} P, P$ has nonempty interior. We use the symbol $|S|$ to denote the number of elements in the set $S$. For any covering $\mathfrak{e}=\left\{C_{1}, \cdots, C_{k}\right\}$ of $P$ we define the complexity $\mathfrak{K}(\mathbb{e}, P)$ to be the ordered pair $(r, s)$, where

$$
\begin{aligned}
& r=\mid\left\{j \mid \text { Int } P \cap C_{j} \neq \varnothing\right\} \mid, \\
& s=\mid\left\{S \subset\{1, \cdots, k\} \mid \text { Int } P \cap \bigcap_{j \in S} C_{j} \neq \varnothing\right\} \mid .
\end{aligned}
$$

Further, we establish a linear order among the complexities of all coverings $\mathcal{C}$ of all $n$-dimensional polyhedra $P$ by defining $\mathcal{K}\left(\mathfrak{C}^{\prime}, P^{\prime \prime}\right)$ $=\left(r^{\prime}, s^{\prime}\right) \prec \mathfrak{\kappa}\left(\mathbb{C}^{\prime \prime}, P^{\prime \prime}\right)=\left(r^{\prime \prime}, s^{\prime \prime}\right)$ if

$$
r^{\prime}<r^{\prime \prime}, \text { or } r^{\prime}=r^{\prime \prime}, \quad s^{\prime}<s^{\prime \prime} .
$$

Observe that $r=1$ implies $s=1$. For $\mathscr{K}(\mathfrak{C}, P)=(1,1)$, the theorem is obvious, for if Int $P \subset C_{j}$ for some $j$, then $P=C_{j}$ because $C_{j}$ is closed. We now proceed by induction on $\mathcal{K}(\mathfrak{e}, P)$.

Assume that $\mathcal{K}(\mathcal{e}, P)=(r, s)$, and the theorem has been proved for all preceding complexities. Clearly $r \leqq s \leqq 2^{r}-1, r>1$.

Case 1. Assume $s<2^{r}-1$. Then there exists a nonempty subset $S \subset\{1, \cdots, k\}$ and an index $m$ such that the convex sets $K=\operatorname{Int} P$ $\cap \bigcap_{j \in S} C_{j} \neq \varnothing$ and $L=$ Int $P \cap C_{m} \neq \varnothing$ satisfy $K \cap L=\varnothing$. They can therefore be weakly separated; i.e., there exists a nonzero vector $a$ and a constant $b$ such that

$$
H_{K}=\{x \mid(a, x)>b\} \text { and } H_{L}=\{x \mid(a, x)<b\}
$$

satisfy $H_{K} \cap K=\varnothing, H_{L} \cap L=\varnothing$.

Let $P_{K}=P \cap$ closure of $H_{K}, P_{L}=P \cap$ closure of $H_{L}, \mathfrak{e}_{K}=\left\{C_{1} \cap P_{K}\right.$, $\left.\cdots, C_{k} \cap P_{K}\right\}, \mathfrak{e}_{L}=\left\{C_{1} \cap P_{L}, \cdots, C_{k} \cap P_{L}\right\}$. Let $(r, s)=\mathfrak{K}(\mathbb{e}, P)$, $\left(r^{K}, s^{K}\right)=\mathscr{K}\left(\mathfrak{C}_{K}, P_{K}\right),\left(r^{L}, s^{L}\right)=\mathscr{K}\left(\mathfrak{C}_{L}, P_{L}\right)$. From $(2.3), r^{K} \leqq r, s^{K}<s$ (since Int $P \cap \bigcap_{j \in S} C_{j} \neq \varnothing$, but Int $\left.P_{K} \cap \bigcap_{j \in S}\left(C_{j} \cap P_{k}\right)=\varnothing\right)$ ), and $r^{L}<r$ (since Int $P \cap C_{m} \neq \varnothing$, but Int $P_{L} \cap\left(C_{m} \cap P_{L}\right)=\varnothing$ ). It follows from the induction hypothesis on complexity that there exist polyhedra $P_{K j} \subset C_{j} \cap P_{K} \subset C_{j}, j=1, \cdots, k$ and $P_{L j} \subset C_{j} \cap P_{L} \subset C_{j}, j$ $=1, \cdots, k$ such that $P_{K}=P_{K 1} \cup \cdots \cup P_{K k}, P_{L}=P_{L 1} \cup \cdots \cup P_{L k}$. The theorem now follows from (2.1).

Case 2. Assume $s=2^{r}-1$. Without loss of generality, we may assume $r=k$, and it follows that $D=\bigcap_{j=1}^{k} C_{j} \neq \varnothing$.

Case 2a. Assume $D$ bounded. Let $E$ be any bounded polyhedron (if $P$ is bounded, take $P=E$ ) such that $D \subset E$, and let $x$ be an arbitrary point in $D$. Then $P \cap E$ is a bounded polyhedron with faces $F_{1}, \cdots, F_{f}$. Clearly each $F_{i}=\bigcup_{j=1}^{k} F_{i} \cap C_{j}$. Since $\operatorname{dim} F_{i}<n$, our induction hypothesis on the dimension shows that for each $i$ there 
exist polyhedra $P_{i j}$ such that $P_{i j} \subset F_{i} \cap C_{j} \subset C_{j}, j=1, \cdots, k$, with $F_{i}=\bigcup_{j=1}^{k} P_{i j}$. Because $P \cap E$ is bounded, each point of $P \cap E$ is on a line segment joining $x$ to a face of $P \cap E$. Let $P_{j}$ be the convex hull of $x$ and all $P_{i j}, i=1, \cdots, f$. Then $P \cap E=\cup_{j=1}^{k} P_{j}$ and $P_{j} \subset C_{j}, j$ $=1, \cdots, n$.

Now the closure of $P-(P \cap E)$ is by (2.2) the union of polyhedra, say $Q_{1}, \cdots, Q_{q}$. Each $Q_{i}$ is covered by $\left\{Q_{i} \cap C_{1}, \cdots, Q_{i} \cap C_{k}\right\}$, and the complexity of each such covering of each $Q_{i}$ precedes $\mathcal{K}(\mathfrak{e}, P)$, since $D \cap$ Int $Q_{i}=\varnothing$ for each $i$. Thus the induction hypothesis on complexity proves the theorem for each $Q_{i}$ and we now apply (2.1).

Case $2 \mathrm{~b}$. Assume $D$ unbounded. Then $D$ contains a ray i.e., there is a point $x$ and a vector $t$ such that $x+\lambda t \in D$ for all $\lambda \geqq 0$. Call this ray $R$ and note that $R$ is a polyhedron contained in each $C_{j}$. Since $D \subset P$ and $P$ is in the first orthant, it follows that

$$
\begin{array}{rl}
x_{i} \geqq 0 & i=1, \cdots, n . \\
t_{i} \geqq 0 & i=1, \cdots, n, \quad \text { at least one } t_{i}>0 .
\end{array}
$$

bdry $P$ is nonempty.

By the induction hypothesis on dimension, if $F_{1}, \cdots, F_{f}$ are the faces of $P$, there exist polyhedra $P_{i j}, i=1, \cdots, f ; j=1, \cdots, k$, such that $P_{i j} \subset C_{j}, j=1, \cdots, k, \cup_{j=1}^{k} P_{i j}=F_{i}$. Let $P_{j}$ be the convex hull of $R$ and all $P_{i j}, i=1, \cdots, n$. Clearly $P_{j} \subset C_{j}$, and all we need show is that every point of $P$ is on a line segment joining a point of $R$ with a point on bdry $P$.

Let $y=\left(y_{1}, \cdots, y_{n}\right) \in P$. From (2.5) there exists a point $r$ $=\left(r_{1}, \cdots, r_{n}\right) \in R$ and an index $i_{0}$ such that $r_{i_{0}}>y_{i_{0}}$.

Consider the point $y(\alpha)=(1 /(1-\alpha))\left(y_{1}-\alpha r_{1}, \cdots, y_{n}-\alpha r_{n}\right)$, which is a continuous function of $\alpha$. When $\alpha=0, y(\alpha)=y$. When $\alpha$ is close to 1 , since $r_{i_{0}}>y_{i_{0}}, y(\alpha)$ is outside the first orthant, hence outside $P$. Hence there is a number $\alpha_{0}, 0 \leqq \alpha_{0}<1$ such that $y\left(\alpha_{0}\right) \in$ bdry $P$. Thus

$$
y=\left(1-\alpha_{0}\right) y\left(\alpha_{0}\right)+\alpha_{0} r,
$$

which is the result desired.

3. An application. Let $n>m$, and let $C_{1}, \cdots, C_{n}$ be closed convex pointed cones in $R^{n}$. For any $n \times m$ real matrix $A$, denote the rows of $A$ by $A_{1}^{\prime}, \cdots, A_{n}^{\prime}$. For any cone $C$ and any vector $x$, the expression $x^{\prime} C>0$ means that $x$ makes a positive inner product with every nonzero $y \in C$. In an investigation of systems of strong linear inequalities on the rows of an $n \times m$ matrix which imply that the matrix is of full rank, the equivalence of the following two statements was proved [1]: 


$$
\begin{aligned}
& A_{i}^{\prime} C_{i}>0 \quad \text { for all } i \text { implies rank } A=m \\
& \bigcup_{i=1}^{n} C_{i} \cup \bigcup_{i=1}^{n}-C_{i}=R^{m} .
\end{aligned}
$$

Because of the potential usefulness of (3.1) in numerical work, it is of some interest to find families of cones $\left\{C_{i}\right\}$ satisfying (3.2) such that if cones $D_{i} \subset C_{i}$ also satisfy (3.2) then $D_{i}=C_{i}$. The reason is that "smaller" cones prove more matrices to be of full rank. That such minimal cones exist is an easy consequence of Zorn's lemma, as Alex Heller has kindly pointed out to us (partial order the families of cones satisfying (3.2) by respective inclusion, choose a maximal simply ordered subset and form intersections). We now show that such a minimal family of cones is polyhedral. Assume $\left\{C_{\boldsymbol{i}}\right\}$ satisfying (3.2). Since $\cup C_{i} \cup \cup-C_{i}=R^{m}$, and $R^{m}$ is a polyhedron, there exist polyhedra $P_{i}, i= \pm 1, \cdots, \pm n$, such that $P_{i} \subset C_{i}, i=1, \cdots, n, P_{-i}$ $C-C_{i}, i=-1, \cdots,-n$, and $\bigcup_{i=1}^{n} P_{i} \cup \bigcup_{i=-1}^{-n} P_{i}=R^{m}$. Let $E_{i}$ be the polyhedral cone spanned by $P_{i}$ and $-P_{-i}, i=1, \cdots, n$. Then $E_{i} \subset C_{i}, i=1, \cdots, n$ and $\cup E_{i} \cup \mathrm{U}-E_{i}=R^{m}$, since $P_{i} \subset E_{i}, P_{-1} \subset-E_{i}$, $i=1, \cdots, n$.

4. Remark. We conjecture (but have been unable to prove) the following: Let $C_{1}, \cdots, C_{k}$ be closed convex subsets of a bounded polyhedron $P$, and let $0<t$ be an integer such that every $t$-flat which meets $P$ meets $\cup_{i=1}^{k} C_{i}$; then there exist polyhedra $P_{i} \subset C_{i}, i=1, \cdots, k$ such that every $t$-flat which meets $P$ meets $\cup P_{i}$.

\section{REFERENCES}

1. A. J. Hoffman, On unions and intersections of cones, Proceedings of the Third Waterloo Conference on Combinatorial Mathematics (to appear).

ibM, Thomas J. Watson Rrsearch Center, Yorktown Heights, New York 